\title{
Dyslipidemia Prevalence among Tinnitus Patients of a Referral ENT Clinic in Tehran, Iran, 2018
}

\author{
Kourosh Etemadi ${ }^{1}$, Ali Goljanian Tabrizi², Mahdieh Fahimi ${ }^{3 *}$ \\ ${ }^{1}$ Department of Epidemiology, School of Public Health and Safety \& Environmental and Occupational Hazards Control \\ Research Center, Shahid Beheshti University of Medical Sciences, Tehran, Iran \\ 2ENT Department, Taleghani Hospital, Shahid Beheshti University of Medical Sciences, Tehran, Iran \\ ${ }^{3}$ School of Health and Safety, Shahid Beheshti University of Medical Sciences, Tehran, Iran
}

\begin{abstract}
Background: Tinnitus is a common neurosensory disorder that can bring many problems such as stress and sleep disorders to the patients and affect their quality of life. Unfortunately, its pathophysiology has not been exactly discovered yet, but there have been some studies executed about the possibility of dyslipidemia causing tinnitus. Studying more on this subject and especially on the Iranian population in order to provide us with evidence-based information, can help us find the optimal method to control and treat this disorder.

Methods: In this study, 76 patients that have had complaints of tinnitus in the ENT (ears, nose, and throat) clinic of Taleghani hospital, Shahid Beheshti University of Medical Sciences, in the year 2018, were selected. Also, after taking a history about the severity and the onset of their problem, they were examined and also blood samples were taken to check their lipid profiles. Finally, the results of the lab tests, the severity and the onset of their disorder and also their age and sex were statistically analyzed. Results: The study showed that the prevalence of dyslipidemia in patients with tinnitus is not significantly greater than its prevalence in the general population. Furthermore despite the relationship between dyslipidemia and the onset and severity of the tinnitus is not significant, the effect of dyslipidemia on tinnitus shows itself after about one year and a half $(P=0.000)$, it means dyslipidemia does not cause the tinnitus but affects in its duration. It also indicated the most common type of dyslipidemia among the patients that we studied, was Hypercholesterolemia with the prevalence of $23.68 \%$.

Conclusion: The Study suggests that despite the Hypercholesterolemia prevalence of $23.68 \%$ among these patients, dyslipidemia does not have a significant relationship with the severity of the neurosensory disorder "tinnitus' overall, but dyslipidemia affects tinnitus after suffering for about one year and a half. Therefore there will not be a need to use blood tests in order to measure the lipid profile factors at the beginning and the first months of the patients' tinnitus.

Keywords: Prevalence; Tinnitus; Dyslipidemia.
\end{abstract}

Citation: Etemadi K, Goljanian Tabrizi A, Fahimi M. Dyslipidemia prevalence among tinnitus patients of a referral ENT clinic in Tehran, Iran, 2018. Int Clin Neurosci J. 2019;6(2):59-63. doi:10.15171/icnj.2019.12

\author{
*Correspondence to \\ Mahdieh Fahimi, Master \\ Student of MPH, School of \\ Health and Safety, Shahid \\ Beheshti University of \\ Medical Sciences, Tehran, \\ Iran. \\ Tel: +989126971654 \\ Email: \\ mahdieh.fahimi@gmail.com
}

Published online 29 June 2019

\section{Introduction}

Tinnitus is the common neurosensory disorder of perceiving a non-verbal noise like ringing without any actual source related to it. This disorder exists in both male and female patients at any age, and it can bring many problems such as difficulties with sleeping, concentration, and psychological problems, therefore affecting their quality of life. Epidemiological studies on the tinnitus prevalence suggest a prevalence of $10 \%$ with no relation to the patients' gender. ${ }^{1}$ Also according to another study in South Korea, this disorder had a prevalence of $20.7 \%$ with $69.2 \%$ of these patients having no complaints of the tinnitus interfering with their lives, $27.9 \%$ having some discomfort, and $3 \%$ having severe problems with it. ${ }^{2}$
Studies suggest that a malfunction in the limbic system and the sympathetic part of the autonomic nervous system due to the false signals of tinnitus bring problems such as anxiety, concentration difficulties, and panic attacks. ${ }^{3}$ The pathophysiology of tinnitus is indeed complicated and not yet determined; nonetheless, data are suggesting a sort of interaction between the peripheral and central pathologies of the auditory system. ${ }^{4}$

There has been an increasing number of studies and clinical trials since the mid-1800s which show a sort of malfunction in the cochleovestibular system in the presence of hypertension, Diabetes Mellitus, and dyslipidemia..$^{5-7}$ Tinnitus can be due to the damage of the hairy cells of the inner ear, and it may all be the result 
of some metabolic disorders such as hyperinsulinemia, dyslipidemia and Diabetes Mellitus. ${ }^{8,9}$ Spencer was the first researcher to discover the relationship between dyslipidemia and tinnitus. He found out that many tinnitus patients, were also predisposed to obesity, maturity-onset diabetes of the young, atherosclerosis and coronary artery disease and all these conditions have a common risk factor which is dyslipidemia. ${ }^{10}$ In a study by Kazmierczak and Doroszewska, the relationship between tinnitus and the glucose metabolism markers were reported significant, but the lipid metabolism markers were not significantly different from the control group. ${ }^{11}$ In another study by Cooper on health and nutrition, the prevalence of dyslipidemia in tinnitus patients was not significantly different from the general population. ${ }^{12}$ There was also another study in Tehran by Anbari et al that showed no significant relationship between dyslipidemia and tinnitus or neurosensory deafness among patients of 5 to 18 years of age. ${ }^{13}$ In a cross-sectional study by Shirazi et al in Iran, the most common impaired element in a lipid profile was cholesterol with a prevalence of $14.4 \%$ and after that low HDL (12.8\%). ${ }^{14}$ However, the relationship between dyslipidemia and tinnitus was not significant.

On the other hand, a study by Basut et al suggested that a low carbohydrate and low lipid diet can decrease the severity of tinnitus. ${ }^{15}$ Also, a study by Arnold et al on a 169 patient sample ${ }^{16}$ and another study by Qian et al on 185 perimenopause women that studied how Chinese medicine can affect the side effects of menopause, ${ }^{17}$ approve of the relationship between dyslipidemia and tinnitus. In a study by Pulec et al the prevalence of dyslipidemia among tinnitus patients was reported 5.1\%, and that the tinnitus could alleviate after a low lipid diet. ${ }^{18}$ A novel study by Evans et al was done to assess the auditory function and dyslipidemia, ${ }^{19}$ which suggested that high levels of triglyceride can bring neurosensory hearing impairments, but a temporary diet of high levels of lipid does not have much effect on the auditory system.

Considering the studies mentioned above and the very different results from them, there is an absolute need to design and carry out more studies in order to find the answer to the same question as these mentioned studies, but this time in Iranian population. Because having the result can help us decide on the right approach and ask for the right lab tests, which can ease the process of curing the tinnitus, and therefore it will increase the patients' quality of life and lower the expenses.

\section{Methods}

In this cross-sectional study, the target population was the patients who suffer from tinnitus, and the source population was the patients with idiopathic tinnitus who are visited by the ENT specialists in the ENT clinic in Talghani hospital, Shahid Beheshti University of Medical Sciences in the year 1397. The inclusion criteria were as follows: the study population in this study was the patients with tinnitus who came for a visit with the ENT specialists in the ENT clinic of Taleghani hospital, Shahid Beheshti University of Medical Sciences in the year 1397 and they had suffered from the tinnitus for at least six months. Also, those patients with the characteristics mentioned below are excluded from the study.

- Any patient with the age of less than 20 or more than 90 years

- Any history of trauma to the head and neck in the last two years before the onset of their tinnitus

- Any patient suffering from the pulsatile tinnitus, objective tinnitus, or somatosounds

- Any sign indicating that the patient is malingering or exaggerating in the description of the tinnitus and the auditory symptoms

The sampling method in this study was convenience sampling. So we did the sampling from among the patients whose diagnosis of tinnitus were already approved by the ENT specialists in our ENT clinic, and also met the inclusion criteria and did not satisfy the exclusion ones. To calculate the sample size we used the results of the study done by Pulec et al which showed the prevalence of dyslipidemia in tinnitus patients $5.1 \%$. Thus the sample size of our study was calculated, 74 patients. Finally, 76 patients entered our study and were then analyzed.

In this study, at first, we took medical histories of the patients of the sample group to determine the onset of their tinnitus and its severity. Then we took blood samples to evaluate their lipid profiles in the laboratory of Taleghani hospital. Finally, the data were analyzed by the software SPSS version 20. We also used this software for the tables and graphs. Linear and ordinal regressions and one-way ANOVA analyses are used in this study. In all the analysis, $P$ values less than 0.05 were considered significant.

\section{Results}

In this study, we assessed 76 patients with idiopathic tinnitus. $52.6 \%$ of them were males, and $47.4 \%$ were females. The mean of age among these 76 people was $64.50 \pm 13.74$, which was $63.87 \pm 13.96$ among males and $65.19 \pm 13.65$ among females.

The time that passed from the onset of tinnitus (according to the patients' medical history) was six months in $46 \%$ of the patients, one year in $17 \%, 1.5$ years in $11 \%$ and it was two years in 9 patients $11.8 \%$. The severity of the tinnitus (according to the patients' medical history) was mild in $26.3 \%$ of the patients, moderate in $48.7 \%$ and severe in $25 \%$. The mean of serum cholesterol among all the patients, and among males and females separately was $226.76 \pm 81.51, \quad 238.03 \pm 93.81$ and $214.65 \pm 64.21$ respectively. The mean of serum cholesterol among all the patients, and among males and females separately was $169.22 \pm 82.66, \quad 168.96 \pm 92.31$ and $169.50 \pm 71.72$ respectively. The mean of serum HDL among all the patients, and among males and females separately was 
$50.76 \pm 10.60,46.45 \pm 9.74$ and $55.56 \pm 9.49$ respectively. The mean of serum LDL among all the patients, and among males and females separately was $125.62 \pm 17.54$, $123.75 \pm 16.22$ and $127.69 \pm 18.91$ respectively. The differences between the male and the female groups were shown to be not significant.

The data has analyzed through linear regression to assess the effect of the patients' age on the severity and onset of their tinnitus. The p-value of the relationship between age and severity was 0.708 which showed age has no significant effect on severity. The $P$ value of the relationship between age and onset was calculated 0.024 , which showed the significant effect of age on the time that it takes for the patients to complain to a specialist after their problem has started. So the younger the patient, the less time they tolerate the disorder.

To study the effect of serum cholesterol, triglyceride, HDL and LDL level on severity and the time passed from the onset of the tinnitus, we analyzed the data by the use of linear regression. This analysis showed a significance of $0.647,0.534,0.053$ (in females) and 0.304 (in males), and 0.400 for severity and $0.811,0.334,0.824$ (in females) and 1.000 (in males), and 0.776 for the onset respectively, ergo not significant for any of the two dependent variables.

There were also results from another series of analysis. It was according to the dual entity of the variable "onset" which in this study is quantitative, but can also be an ordinal one since the amounts have only given as the exact amounts of $0.5,1,1.5$ and 2 in this variable group. Thus we analyzed them through ordinal regression as well. In the analysis by the use of the ordinal regression on the effect of serum cholesterol, triglyceride, HDL and LDL level on the onset of the tinnitus, the significance for the onset of $0.5,1$ and 1.5 years was calculated $0.567,0.075$ and 0.000 respectively about cholesterol, $0.701,0.007$ and 0.000 for triglyceride, $0.948,0.026$ and 0.000 for HDL in females and $0.381,0.007$ and 0.000 for HDL in males, and $0.717,0.057$ and 0.000 about LDL. These numbers explain that the elements of the lipid profile are not the factors that affect tinnitus even until 6 months, but after about one year or one year and a half passed with suffering from this neurosensory disorder, the effects of the lipid profile components start to appear.

To study the effect of sex on severity and onset of the tinnitus, we used the one-way ANOVA analysis which brings the p-values of 0.881 and 0.650 respectively. And to study the effect of the severity on the onset and onset of the tinnitus, we also used the one-way ANOVA analysis which brings the p-value of 0.041 which is significant and shows that the more severe the tinnitus, the sooner the patient needs the urge to visit the ENT specialist and complain about his or her problem.

\section{Discussion}

The most prevalent type of dyslipidemia among the patients we studied in this study was revealed to be hypercholesterolemia with a prevalence of $23.68 \%$. The second most prevalent one was a high level of serum triglyceride (in 11.84\%) after that was low HDL level (9.21\%) and finally, LDL which was abnormal only in $5.26 \%$ of the patients of our study sample. However, these were not significantly higher than the general population.

This study showed no significant relationship between sex and the two variables of severity and onset, and also between age and severity. However, age does affect the duration from the onset until the patient needs an ENT visit, in the way that the younger the patient, the sooner they complain about their disorder.

The statistical analysis showed that the relationship between each of the lipid profile elements with the two variables of severity and onset of tinnitus is not significant in the analysis of all the patients together. Also, the prevalence of dyslipidemia among the patients with tinnitus, whom we studied, was not significantly higher than its prevalence among the general population. On the other hand, the calculations revealed that all the abnormal amounts of all the lipid profile elements started to show their effect after about one year and a half from the onset of the tinnitus. This effect was so significant with a $P$ value of less than 0.001; triglyceride and HDL in both our male and female patients started to affect the tinnitus even after only one year. So the reason that the relationship between dyslipidemia and onset of the tinnitus has not concluded as significant in the first part of the analysis was that we had analyzed all the patients together as one group, whether they were suffering tinnitus for only six months or they had it for two years! However, in the second part of the analysis when we categorized the patients according to the onset of their disorder, the effect of dyslipidemia became utterly significant. The important point to explain this result is that the lipid profile elements do not affect the incidence of tinnitus and its first months of disturbing the patients' hearing; but their effect is absolutely not deniable after the periods of time passed from the onset that were mentioned above according to each of the lipid elements. In other words dyslipidemia does not cause the tinnitus, but has a significant direct relationship with it that emerges in the duration of the disease, which acts on tinnitus alongside the other factors that are responsible for causing the disease in the first place or worsening it.

To explain this result of insignificance we could mention the effect of the patients' dietary routines that could have affected the blood test results since these patients were from different age groups, so according to their different age and different past medical histories, they would have had different dietary routines with different lipid content. Also, another reason to this result is the difference in the patient's drug histories and the medication that these patients had been already taking in order to control and correct their lipid profile abnormalities even before their tinnitus started.

The result of this study supports the ones from the 
studies done by Shirazi et al, ${ }^{14}$ Anbari et al, ${ }^{13}$ Cooper, ${ }^{12}$ Kazmierczak and Doroszewska, ${ }^{11}$ that show no significant relationship between dyslipidemia and tinnitus overall. Although the prevalence of dyslipidemia is evaluated higher than that in the study of Shirazi et al. ${ }^{17}$ Also, the second most prevalent type of dyslipidemia in our study was high serum level of triglyceride, while it was low HDL serum levels in the study of Shirazi et al. Furthermore our study showed different results than the other studies mentioned in the literature review, which we explain one by one below:

In the study by Basut et al on 52 patients in Turkey, a lowcarb and low-fat diet had been effective in the reduction of the severity of tinnitus. ${ }^{15}$ To explain their result we could point out the fact that this reduction in severity could be due to the reduction in the carbohydrates of the patients' diet, but our study had not evaluated the role of a diet change in the matter. There were also another two studies which indicated a relationship between tinnitus and dyslipidemia. These were the study of Arnold et $\mathrm{al}^{16}$ (on a 169 patient sample) and the study done by Qian et al (on 185 perimenopause women to assess the effect of Chinese medicine on menopause side effects. ${ }^{17}$ The difference between our results and theirs could be due to their study samples which contain patients with other medical conditions which can confound the results and make it hard for us to compare the results of these studies to each other. In the study of Pulec et $\mathrm{al}^{18}$ in California which studied 2332 patients to assess the effects of dyslipidemia on neurosensory hearing loss, dizziness and tinnitus, the prevalence of dyslipidemia among patients with tinnitus was reported $21 \%$ which is not significantly higher than the general population, although Pulec et al had shown that these problems could alleviate by the use of a lowfat diet. This is while we evaluated the dyslipidemia prevalence among tinnitus patients higher than Pulec's study. Now we finally explain about the result of the study done by Evans et $\mathrm{al}^{19}$ in Huston, USA. It was done in the year 2013 and was carried out in two parts, one on humans and one on animal samples, that in the human part, showed a significant relationship between increased levels of triglyceride and a decrease in auditory functions, but reported no significant relationship between auditory functions with the serum level of HDL and LDL. So about these results we could that say the auditory functions may not include tinnitus, ergo the study cannot prove the effect of dyslipidemia on this disorder.

Furthermore, none of the studies mentioned above takes the variable "onset" into account, but our study does it which shows the effects of the lipid profile factors start from the time about a year or a year and a half after the onset of their disorder. Although this study does not show a significant difference in dyslipidemia prevalence between tinnitus and the general population, and it also indicates that these lipid abnormalities have no significant effect on the severity of tinnitus, the higher prevalence of hypercholesterolemia than the other types of dyslipidemia among these patients can be a clue for designing some further research.

\section{Conclusion}

This study finally showed that dyslipidemia in patients with tinnitus is not overall significantly higher than in the general population and does not affect the severity of tinnitus in the patients. However, their effects get more distinct and also detectable after about one year and a half from the onset of this neurosensory disorder. It means that before this time, there are other factors than dyslipidemia that cause tinnitus. Therefore the question that asks if we could decrease the incidence and the severity of tinnitus by correcting their lipid profile elements through controlling the patients' diet and prescribing special medication does not get a positive answer yet. Thus it is our job to design more studies to search for factors affecting tinnitus other than just dyslipidemia. Therefore we might be able to improve the quality of life in patients suffering from tinnitus, lower the number of these patients, lower the number of their visits in the clinic and also decrease the time and expenses that we spend nowadays to treat this disorder.

\section{Conflict of Interest Disclosures}

The authors declare that they have no conflict of interests.

\section{Ethical Statement}

The ethical and humane considerations in this study are approved by the research ethics committee of Shahid Beheshti University of Medical Sciences. In this study we explained the process to the patients completely and then they entered the study voluntarily and with informed consent. Confidentiality, privacy and secrecy were completely considered and respected in all the steps of our study.

No burden of any kind of harming or monetary expenses was loaded on the patients of the sample. The tinnitus patients that were detected to have dyslipidemia in their lab results were referred to internists to start the respective treatments. Finally, ethical codes and rules were considered in the use of the references and former studies and also in publishing the results of this study.

\section{References}

1. Baguley D, McFerran D, Hall D. Tinnitus. Lancet. 2013;382(9904):1600-7. doi: 10.1016/s01406736(13)60142-7.

2. Huang YS, Koo M, Chen JC, Hwang JH. The association between tinnitus and the risk of ischemic cerebrovascular disease in young and middle-aged patients: A secondary case-control analysis of a nationwide, population-based health claims database. PLoS One. 2017;12(11):e0187474. doi: 10.1371/journal.pone.0187474.

3. Jastreboff PJ, Jastreboff MM. Tinnitus retraining therapy: a different view on tinnitus. ORL J Otorhinolaryngol Relat Spec. 2006;68(1):23-9; discussion 9-30. doi: 10.1159/000090487.

4. Norena AJ, Farley BJ. Tinnitus-related neural activity: theories of generation, propagation, and centralization. Hear Res. 2013;295:161-71. doi: 10.1016/j.heares.2012.09.010.

5. de Moraes Marchiori LL, de Almeida Rego Filho E, Matsuo T. Hypertension as a factor associated with hearing loss. Braz J 
Otorhinolaryngol. 2006;72(4):533-40.

6. Perez R, Ziv E, Freeman S, Sichel JY, Sohmer H. Vestibular end-organ impairment in an animal model of type 2 diabetes mellitus. Laryngoscope. 2001;111(1):110-3. doi: 10.1097/00005537-200101000-00019.

7. Tachibana M, Yamamichi I, Nakae S, Hirasugi Y, Machino $M$, Mizukoshi O. The site of involvement of hypertension within the cochlea. A comparative study of normotensive and spontaneously hypertensive rats. Acta Otolaryngol. 1984;97(3-4):257-65.

8. Holcat M. [Tinnitus and diabetes]. Vnitr Lek. 2007;53(5):5346.

9. Wang H, Tian J, Yin D, Jiang S, Yang W, Han D, et al. Regional glucose metabolic increases in left auditory cortex in tinnitus patients: a preliminary study with positron emission tomography. Chin Med J (Engl). 2001;114(8):848-51.

10. Spencer JT Jr. Hyperlipoproteinemia and inner ear disease. W V Med J. 1974;70(9):215-21.

11. Kazmierczak H, Doroszewska G. Metabolic disorders in vertigo, tinnitus, and hearing loss. Int Tinnitus J. 2001;7(1):548.

12. Cooper JC Jr. Health and Nutrition Examination Survey of 1971-75: Part II. Tinnitus, subjective hearing loss, and wellbeing. J Am Acad Audiol. 1994;5(1):37-43.

13. Anbari S, Isazadeh D, Safavi A, Alaie M, Azizi F. The role of dyslipidemia in sensorineural hearing loss in children. Int J Pediatr Otorhinolaryngol. 2010;74(1):32-6. doi: 10.1016/j. ijporl.2009.10.003

14. M-Shirazi M, Farhadi M, Jalessi M, Kamrava SK, HeshmatzadehBehzadi A, Arami B. Prevalence of dyslipidemia among Iranian patients with idiopathic tinnitus. J Res Med Sci. 2011;16(7):890-6.

15. Basut O, Ozdilek T, Coskun H, Erisen L, Tezel I, Onart S, et al. [The incidence of hyperinsulinemia in patients with tinnitus and the effect of a diabetic diet on tinnitus]. Kulak Burun Bogaz Ihtis Derg. 2003;10(5):183-7.

16. Arnold M, Bousser MG, Fahrni G, Fischer $U$, Georgiadis D, Gandjour J, et al. Vertebral artery dissection: presenting findings and predictors of outcome. Stroke. 2006;37(10):2499503. doi: 10.1161/01.str.0000240493.88473.39.

17. Qian LQ, Wang B, Niu JY, Gao S, Zhao DY. Assessment of the clinical effect of Chinese medicine therapy combined with psychological intervention for treatment of patients of perimenopausal syndrome complicated with hyperlipidemia. Chin J Integr Med. 2010;16(2):124-30. doi: 10.1007/s11655010-0124-x.

18. Pulec JL, Pulec MB, Mendoza I. Progressive sensorineural hearing loss, subjective tinnitus and vertigo caused by elevated blood lipids. Ear Nose Throat J. 1997;76(10):716-20, 25-6, 28 passim.

19. Evans MB, Tonini R, Shope $C D$, Oghalai JS, Jerger JF, Insull $\mathrm{W}$ Jr, et al. Dyslipidemia and auditory function. Otol Neurotol. 2006;27(5):609-14. doi: 10.1097/01. mao.0000226286.19295.3 